\title{
Editorial
}

\section{Artificial Intelligence and Its Applications}

\author{
Yudong Zhang, ${ }^{1}$ Saeed Balochian, ${ }^{2}$ Praveen Agarwal, ${ }^{3}$ \\ Vishal Bhatnagar, ${ }^{4}$ and Orwa Jaber Housheya ${ }^{5}$ \\ ${ }^{1}$ School of Computer Science and Technology, Nanjing Normal University, Nanjing, Jiangsu 210023, China \\ ${ }^{2}$ Department of Electrical Engineering, Gonabad Branch, Islamic Azad University, Gonabad, Khorasan-e-Razavi 96916-29, Iran \\ ${ }^{3}$ Department of Mathematics, Anand International College of Engineering, Agra Road, Near Bassi, Jaipur, Rajasthan 303012, India \\ ${ }^{4}$ Ambedkar Institute of Advanced Communication Technologies and Research, Government of NCT of Delhi, \\ Geeta Colony, Delhi 110031, India \\ ${ }^{5}$ Department of Chemistry, Arab American University, 19356 Jenin, Palestine
}

Correspondence should be addressed to Yudong Zhang; zhangyudongnuaa@gmail.com

Received 18 March 2014; Accepted 18 March 2014; Published 10 April 2014

Copyright (c) 2014 Yudong Zhang et al. This is an open access article distributed under the Creative Commons Attribution License, which permits unrestricted use, distribution, and reproduction in any medium, provided the original work is properly cited.

Artificial intelligence (AI) has revolutionized information technology. The new economy of information technology has shaped the way we are living. Recently, AI algorithms have attracted close attention of researchers and have also been applied successfully to solve problems in engineering. Nevertheless, for large and complex problems, AI algorithms consume considerable computation time due to stochastic feature of the search approaches. Therefore, there is a potential requirement to develop efficient algorithm to find solutions under the limited resources, time, and money in realworld applications. This special issue aims to report the latest advances in every aspect of artificial intelligence technology, including machine learning, data mining, computer vision, multiagent systems, evolutionary computation, and fuzzy logic.

The special issue received 142 high quality submissions from different countries all over the world. All submitted papers followed the same standard (peer-reviewed by at least three independent reviewers) as applied to regular submissions to Mathematical Problems in Engineering. Due to the limited space, 57 papers were finally included. The primary guideline was to demonstrate the most significant developments on the topics of AI and to apply AI algorithms in real-life scenarios.

In the paper entitled "Solving two-dimensional HP model by firefly algorithm and simplified energy function," Y. Zhang et al. investigate traditional energy function and point out that its discrete property cannot give direction of the next step to the searching point, causing a challenge to optimization algorithms. Therefore, they introduce the simplified energy function to turn traditional discrete energy function to a continuous one. The simplified energy function totals the distance between all pairs of hydrophobic amino acids. To optimize the simplified energy function, they introduce the latest swarm intelligence algorithm, the firefly algorithm (FA). The experiments take 14 sequences of different chain lengths from 18 to 100 as the dataset and compare the FA with standard genetic algorithm and immune genetic algorithm. Each algorithm runs 20 times. The averaged energy convergence results show that FA achieves the lowest values. It concludes that it is effective to solve $2 \mathrm{D}$ HP model by the FA and the simplified energy function.

In the paper entitled "Fault diagnosis for wireless sensor by twin support vector machine," $\mathrm{M}$. Ding et al. propose a novel fault diagnosis method for wireless sensor technology by twin support vector machine (TSVM) in order to improve the diagnosis accuracy of wireless sensor. Twin SVM is a binary classifier that performs classification by using two nonparallel hyperplanes instead of the single hyperplane used in the classical SVM. However, the parameter setting in the TSVM training procedure significantly influences the classification accuracy. Their study introduces PSO as an optimization technique to simultaneously optimize the TSVM training parameter. The experimental results indicate 
that the diagnosis results for wireless sensor of twin support vector machine are better than those of SVM, ANN.

In the paper "Design of special impacting filter for multicarrier ABPSK system," Z. Chen and L. Wu propose an iterative scheme to derive the parameters of the cascade notch filter. The cost function is determined by the cascading notch filter's influence on impacting filters, converting the cost function's least square problem to a filter parameters' standard quadratic programming problem. Finally, a cascading notch SIF (CNSIF) designed to demodulate the ABPSK signals is realized.

In the paper "Weight-selected attribute bagging for credit scoring," J. Li et al. propose an improved attribute bagging method, weight-selected attribute bagging (WSAB), to evaluate credit risk. Weights of attributes are first computed using attribute evaluation method such as linear support vector machine (LSVM) and principal component analysis (PCA). Subsets of attributes are then constructed according to weights of attributes. For each of attribute subsets, the larger the weights of the attributes, the larger the probabilities by which they are selected into the attribute subset. Next, training samples and test samples are projected onto each attribute subset, respectively. A scoring model is then constructed based on each set of newly produced training samples. Finally, all scoring models are used to vote for test instances. An individual model that only uses selected attributes will be more accurate because of elimination of some of redundant and uninformative attributes. Besides, the way of selecting attributes by probability can also guarantee the diversity of scoring models. Experimental results based on two credit benchmark databases show that the proposed method, WSAB, is outstanding in both prediction accuracy and stability, as compared to analogous methods.

In the paper entitled "A novel method for surface defect detection of photovoltaic module based on independent component analysis," the research of X. Zhang et al. proposes a new method for surface defect detection of photovoltaic module based on independent component analysis (ICA) reconstruction algorithm. Firstly, a faultless image is used as the training image. The demixing matrix and corresponding ICs are obtained by applying the ICA in the training image. Then they reorder the ICs according to the range values and reform the demixing matrix. The reformed demixing matrix is used to reconstruct the defect image. The resulting image can remove the background structures and enhance the local anomalies. Experimental results show that the proposed method can effectively detect the presence of defects in periodically patterned surfaces.

In the paper entitled "Neural model with particle swarm optimization Kalman learning for forecasting in smart grids," A. Y. Alanis et al. discuss a novel training algorithm for a neural network architecture applied to time series prediction with smart grids applications. The proposed training algorithm is based on an extended Kalman filter (EKF) improved using particle swarm optimization (PSO) to compute the design parameters. The EKF-PSO-based algorithm is employed to update the synaptic weights of the neural network. The size of the regression vector is determined by means of the Cao methodology. The proposed structure captures more efficiently the complex nature of the wind speed, energy generation, and electrical load demand time series that are constantly monitored in a smart grid benchmark. The proposed model is trained and tested using real data values in order to show the applicability of the proposed scheme.

In the paper entitled "A wavelet-based robust relevance vector machine based on sensor data scheduling control for modeling mine gas gushing forecasting on virtual environment," W. Ting et al. present a wavelet-based robust relevance vector machine based on sensor data scheduling control for modeling mine gas gushing forecasting. Morlet wavelet function can be used as the kernel function of robust relevance vector machine. Mean percentage error has been used to measure the performance of the proposed method in this study. As the mean prediction error of mine gas gushing of the WRRVM model is less than $1.5 \%$ and the mean prediction error of mine gas gushing of the RVM model is more than $2.5 \%$, it can be seen that the prediction accuracy for mine gas gushing of the WRRVM model is better than that of the RVM model.

In the paper entitled "Practical speech emotion recognition based on online learning: from acted data to elicited data," C. Huang et al. study the cross-database speech emotion recognition based on online learning. How to apply a classifier trained on acted data to naturalistic data, such as elicited data, remains a major challenge in today's speech emotion recognition system. They introduce three types of different data sources: first, a basic speech emotion dataset collected from acted speech by professional actors and actresses; second, a speaker-independent data set which contains a large number of speakers; third, an elicited speech data set collected from a cognitive task. Acoustic features are extracted from emotional utterances and evaluated by using maximal information coefficient (MIC). A baseline valence and arousal classifier is designed based on Gaussian mixture models. Online training module is implemented by using AdaBoost. While the offline recognizer is trained on the acted data, the online testing data includes the speakerindependent data and the elicited data. Experimental results show that by introducing the online learning module their speech emotion recognition system can be better adapted to new data, which is an important character in real world applications.

In the paper entitled "Visual object tracking based on 2DPCA and ML," M.-X. Jiang et al. present a novel visual object tracking algorithm based on two-dimensional principal component analysis (2DPCA) and maximum likelihood estimation (MLE). Firstly, they introduce regularization into the 2DPCA reconstruction and develop an iterative algorithm to represent an object by 2DPCA bases. Secondly, the model of sparsity constrained MLE is established. Abnormal pixels in the samples will be assigned with low weights to reduce the effects on the tracking algorithm. The object tracking results are obtained by using Bayesian maximum a posteriori (MAP) probability estimation. Finally, to further reduce tracking drift, they employ a template update strategy that combines incremental subspace learning and the error matrix. Their strategy adapts the template to the appearance change of the target and reduces the influence of the occluded target template as well. Compared with other popular methods, their 
method reduces the computational complexity and is very robust to abnormal changes. Both qualitative and quantitative evaluations on challenging image sequences demonstrate that the proposed tracking algorithm achieves more favorable performance than several state-of-the-art methods.

In the paper entitled "Artificial bee colony algorithm merged with pheromone communication mechanism for the 01 multidimensional knapsack problem," J. Ji et al. propose a new artificial bee colony (ABC) algorithm for the 0-1 multidimensional knapsack problem (MKP_01). The new ABC algorithm introduces a novel communication mechanism among bees, which bases on the updating and diffusion of inductive pheromone produced by bees. In a number of experiments and comparisons, their approach obtains better quality solutions in shorter time than the $\mathrm{ABC}$ algorithm without the mechanism. They also compare the solution performance of the proposed approach against some stochastic approaches recently reported in the literature. Computational results demonstrate the superiority of the new $\mathrm{ABC}$ approach over all the other approaches.

In another paper, Q. Zhu and L.-N. Wu present "Weighted-bit-flipping-based sequential scheduling decoding algorithms for LDPC codes." Their paper proposes a sequential scheduling algorithm based on weighted bit-flipping (WBF) algorithm for the sake of improving the convergence speed. Notoriously, WBF is a low-complexity and simple algorithm. They combine it with belief propagation (BP) to obtain advantages of these two algorithms. Flipping function used in WBF is borrowed to determine the priority of scheduling. Simulation results show that it can provide a good tradeoff between FER performance and computation complexity for short-length low-density parity-check (LDPC) codes.

In another paper, the research of A. Laudani et al. entitled "CFSO": a new supervised swarm-based optimization algorithm," presents $\mathrm{CFSO}^{3}$, an optimization heuristic within the class of the swarm intelligence, based on a synergy among three different features of the Continuous flock-ofstarlings optimization. One of the main novelties is that this optimizer is no more a classical numerical algorithm since it now can be seen as a continuous dynamic system, which can be treated by using all the mathematical instruments available for managing state equations. In addition, $\mathrm{CFSO}^{3}$ allows passing from stochastic approaches to supervised deterministic ones since the random updating of parameters, a typical feature for numerical swarm-based optimization algorithms, is now fully substituted by a supervised strategy: in $\mathrm{CFSO}^{3}$ the tuning of parameters is a priori designed for obtaining both exploration and exploitation. Indeed the exploration, that is, the escaping from a local minimum, as well as the convergence and the refinement to a solution can be designed simply by managing the eigenvalues of the CFSO state equations. Virtually in $\mathrm{CFSO}^{3}$, just the initial values of positions and velocities of the swarm members have to be randomly assigned. Both standard and parallel versions of $\mathrm{CFSO}^{3}$ together with validations on classical benchmarks are presented.

In the paper "UCAV path planning by fitness-scaling adaptive chaotic particle swarm optimization," Y. Zhang et al. propose a fitness-scaling adaptive chaotic particle swarm optimization (FAC-PSO) approach as a fast and robust approach for the task of path planning of UCAVs. The FAC-PSO employed the fitness-scaling method, the adaptive parameter mechanism, and the chaotic theory. Experiments show that the FAC-PSO is more robust and costs less time than elite genetic algorithm with migration, simulated annealing, and chaotic artificial bee colony. Moreover, the FAC-PSO performs well on the application of dynamic path planning when the threats cruise randomly and on the application of 3D path planning.

In the paper entitled "Semisupervised clustering for networks based on fast affinity propagation," M. Zhu et al. propose a semisupervised clustering algorithm for networks based on fast affinity propagation (SCAN-FAP), which is essentially a kind of similarity metric learning method. Firstly, they define a new constraint similarity measure integrating the structural information and the pairwise constraints, which reflects the effective similarities between nodes in networks. Then, taking the constraint similarities as input, they propose a fast affinity propagation algorithm that keeps the advantages of the original affinity propagation algorithm while increasing the time efficiency by passing only the messages between certain nodes. Finally, by extensive experimental studies, they demonstrate that the proposed algorithm can take full advantage of the prior knowledge and improve the clustering quality significantly. Furthermore, the proposed algorithm has a superior performance to some of the state-of-the-art approaches.

In the paper entitled "Research on the production scheduling optimization for virtual enterprises," M. Huang et al. establish a partner selection model based on an improved ant colony algorithm, then present a production scheduling framework with two layers as global scheduling and local scheduling for virtual enterprise, and give a global scheduling mathematical model with the smallest total production time based on it. An improved genetic algorithm is proposed in the model to solve the time complexity of virtual enterprise production scheduling. The experimental results validate the optimization of the model and the efficiency of the algorithm.

In the paper entitled "Surface defect target identification on copper strip based on adaptive genetic algorithm and feature saliency," X. Zhang et al. propose a new surface defect target identification method for copper strip based on adaptive genetic algorithm (AGA) and feature saliency. First, the study uses gray level cooccurrence matrix (GLCM) and HU invariant moments for feature extraction. Then, adaptive genetic algorithm, which is used for feature selection, is evaluated and discussed. In AGA, total error rates and false alarm rates are integrated to calculate the fitness value, and the probability of crossover and mutation is adjusted dynamically according to the fitness value. At last, the selected features are optimized in accordance with feature saliency and are inputted into a support vector machine (SVM). Furthermore, for comparison, they conduct experiments using the selected optimal feature subsequence (OFS) and the total feature sequence (TFS) separately. The experimental results demonstrate that the proposed method can guarantee 
the correct rates of classification and can lower the false alarm rates.

In the paper entitled "Interesting activities discovery for moving objects based on collaborative filtering," G. Yuan et al. propose a method of interesting activities discovery based on collaborative filtering. First, the interesting degree of the objects' activities is calculated comprehensively. Then, combined with the newly proposed hybrid collaborative filtering, similar objects can be computed and all kinds of interesting activities can be discovered. Finally, potential activities are recommended according to their similar objects. The experimental results show that the method is effective and efficient in finding objects' interesting activities.

In the paper entitled "Optimal design of signal controlled road networks using differential evolution optimization algorithm," H. Ceylan proposes a traffic congestion minimization model in which the traffic signal setting optimization is performed through a combined simulation-optimization model. In this model, the TRANSYT traffic simulation software is combined with differential evolution (DE) optimization algorithm, which is based on the natural selection paradigm. In this context, the equilibrium network design (EQND) problem is formulated as a bilevel programming problem in which the upper level is the minimization of the total network performance index. In the lower level, the traffic assignment problem, which represents the route choice behavior of the road users, is solved using the path flow estimator (PFE) as a stochastic user equilibrium assessment. The solution of the bilevel EQND problem is carried out by the proposed differential evolution and TRANSYT with PFE, the so-called DETRANSPFE model, on a well-known signal controlled test network. Performance of the proposed model is compared to that of two previous works where the EQND problem has been solved by genetic-algorithms- (GAs-) and harmony-search- (HS-) based models. Results show that the DETRANSPFE model outperforms the GA- and HS-based models in terms of the network performance index and the computational time required.

In the paper entitled " $A$ wavelet kernel-based primal twin support vector machine for economic development prediction," F. Su and H. Shang propose an economic development prediction method based on the wavelet kernel-based primal twin support vector machine algorithm. As gross domestic product (GDP) is an important indicator to measure economic development, economic development prediction means GDP prediction in this study. The wavelet kernelbased primal twin support vector machine algorithm can solve two smaller sized quadratic programming problems instead of solving a large one as in the traditional support vector machine algorithm. Economic development data of Anhui province from 1992 to 2009 are used to study the prediction performance of the wavelet kernel-based primal twin support vector machine algorithm. The comparison of mean error of economic development prediction between wavelet kernel-based primal twin support vector machine and traditional support vector machine models trained by the training samples with the 3-5-dimensional input vectors, respectively, is given in this paper. The testing results show that the economic development prediction accuracy of the wavelet kernel-based primal twin support vector machine model is better than that of traditional support vector machine.

In the paper entitled "Multiagent reinforcement learning with regret matching for robot soccer," Q. Liu et al. propose a novel multiagent reinforcement learning (MARL) algorithm Nash-Q learning with regret matching, in which regret matching is used to speed up the well-known MARL algorithm Nash-Q learning. It is of importance to choose the suitable strategy of action selection to balance exploration and exploitation, with the aim of enhancing the ability of online learning of Nash-Q learning. In Markov game the joint action of agents adopting regret matching algorithm can converge to a group of points of no-regret that can be viewed as coarse correlated equilibrium which includes Nash equilibrium in essence. It is can be inferred that regret matching can guide exploration of the state-action space so that the rate of convergence of Nash-Q learning algorithm can be increased. Simulation results on robot soccer validate that compared to original Nash-Q learning algorithm, the use of regret matching during the learning phase of Nash- $Q$ learning has excellent ability of online learning and results in significant performance in terms of scores, average reward, and policy convergence.

In another paper, X. Hu et al. present "Emotion expression of robot with personality." They build a robot emotional expression model based on hidden Markov model (HMM) to enable robots that have different personalities to respond in a more satisfactory emotional level. Gross emotion regulation theory and five factors model (FFM) that are the theoretical basis are firstly described. The importance of the personality effect on the emotion expression process is proposed, and how to make the effect quantization is discussed. After that, the algorithm of HMM is used to describe the process of emotional state transition and expression, and the performance transferring probability affected by personality is calculated. At last, the algorithm model is simulated and applied in a robot platform. The results prove that the emotional expression model can acquire human-like expressions and improve the human-computer interaction.

In another paper, the research of Y.-H. Kim and Y. Yoon entitled "Context prediction of mobile users based on timeinferred pattern networks: a probabilistic approach" presents a probabilistic method of predicting context of mobile users based on their historic context data. The presented method predicts general context based on probability theory through a novel graphical data structure, which is a kind of weighted directed multigraphs. User context data are transformed into the new graphical structure, in which each node represents a context or a combined context and each directed edge indicates a context transfer with the time weight inferred from corresponding time data. They also consider the periodic property of context data and devise a good solution to context data with such property. Test shows the merits of the presented method.

In the paper "Tundish cover flux thickness measurement method and instrumentation based on computer vision in continuous casting tundish," M. Lu et al. specifically design and build instrumentation and present a novel method to 
measure the tundish cover flux (TCF) thickness. The instrumentation is composed of a measurement bar, a mechanical device, a high-definition industrial camera, a Siemens S7200 programmable logic controller (PLC), and a computer. Their measurement method is based on the computer vision algorithms, including image denoising method, monocular range measurement method, scale invariant feature transform (SIFT), and image gray gradient detection method. Using the present instrumentation and method, images in the continuous casting (CC) tundish can be collected by camera and transferred to computer to do image processing. Experiments show that the proposed instrumentation and method worked well at scene of steel plants, can accurately measure the thickness of TCF, and overcome the disadvantages of traditional measurement methods, or even replace the traditional ones.

In the paper entitled "Ripple-spreading network model optimization by genetic algorithm," X.-B. Hu et al. attempt to apply genetic algorithm (GA) to tune the values of ripplespreading related parameters (RSRPs), so that the ripplespreading network model (RSNM) may generate these two most important network topologies. The study demonstrates that, once RSRPs are properly tuned by GA, the RSNM is capable of generating both network topologies and therefore has a great flexibility to study many real-world complex network systems.

In the paper entitled "A hybrid bat algorithm with path relinking for capacitated vehicle routing problem," Y. Zhou et al. propose a hybrid bat algorithm with path relinking (HBA-PR) to solve capacitated vehicle routing problem (CVRP). The HBA-PR is constructed based on the framework of continuous bat algorithm; the greedy randomized adaptive search procedure (GRASP) and path relinking are effectively integrated into bat algorithm. Moreover, in order to further improve the performance, the random subsequences and single-point local search are operated with certain loudness (probability). In order to verity the validity of their method, several classical CVRP instances from three classes of benchmarks are selected to test its efficiency compared with other existing methods. Experimental results and comparisons show that the HBA-PR is effective for CVRP.

In the paper entitled "Study on semi-parametric statistical model of safety monitoring of cracks in concrete dams," C. $\mathrm{Gu}$ et al. consider that cracks are one of the hidden dangers in concrete dams. The study on safety monitoring models of concrete dam cracks has always been difficult. Using the parametric statistical model of safety monitoring of cracks in concrete dams, with the help of the semiparametric statistical theory, and considering the abnormal behaviors of these cracks, the semiparametric statistical model of safety monitoring of concrete dam cracks is established to overcome the limitation of the parametric model in expressing the objective model. Previous projects show that the semiparametric statistical model has a stronger fitting effect and has a better explanation for cracks in concrete dams than the parametric statistical model. However, when used for forecast, the forecast capability of the semiparametric statistical model is equivalent to that of the parametric statistical model. The modeling of the semiparametric statistical model is simple, has a reasonable principle, and has a strong practicality, with a good application prospect in the actual project.

In the paper entitled "Efficient secure multiparty computation protocol for sequencing problem over insecure channel," Y. Sun et al. believe that secure multiparty computation is more and more popular in electronic bidding, anonymous voting, and online auction, as a powerful tool in solving privacy preserving cooperative problems. Privacy preserving sequencing problem that is an essential link is regarded as the core issue in these applications. However, due to the difficulties of solving multiparty privacy preserving sequencing problem, related secure protocol is extremely rare. In order to break this deadlock, their paper presents an efficient secure multiparty computation protocol for the general privacy-preserving sequencing problem based on symmetric homomorphic encryption. The result is of value not only in theory, but also in practice.

In the paper entitled "Nighttime fire/smoke detection system based on a support vector machine," C.-C. Ho states that a laser light can be projected into the monitored field of view and the returning projected light section image can be analyzed to detect fire and/or smoke, in order to overcome the nighttime limitations of video smoke detection methods. If smoke appears within the monitoring zone created from the diffusion or scattering of light in the projected path, the camera sensor receives a corresponding signal. The successive processing steps of the proposed real-time algorithm use the spectral, diffusing, and scattering characteristics of the smoke-filled regions in the image sequences to register the position of possible smoke in a video. Characterization of smoke is carried out by a nonlinear classification method using a support vector machine, and this is applied to identify the potential fire/smoke location. Experimental results in a variety of nighttime conditions demonstrate that the proposed fire/smoke detection method can successfully and reliably detect fires by identifying the location of smoke.

In the paper entitled "Improved SpikeProp for using particle swarm optimization," F. Y. H. Ahmed et al. derive a novel supervised learning rule for SpikeProp to overcome the discontinuities introduced by the spiking thresholding. Their algorithm is based on an error-backpropagation learning rule suited for supervised learning of spiking neurons that use exact spike time coding. The SpikeProp is able to demonstrate the spiking neurons that can perform complex nonlinear classification in fast temporal coding. Their study proposes enhancements of SpikeProp learning algorithm for supervised training of spiking networks which can deal with complex patterns. The proposed methods include the SpikeProp particle swarm optimization (PSO) and angle driven dependency learning rate. These methods are presented to SpikeProp network for multilayer learning enhancement and weights optimization. Input and output patterns are encoded as spike trains of precisely timed spikes, and the network learns to transform the input trains into target output trains. With these enhancements, the proposed methods outperformed other conventional neural network architectures.

In the paper entitled "LGMS-FOA: an improved fruit fly optimization algorithm for solving optimization problems," D. Shan et al. empirically study the performance of fruit 
fly optimization algorithm (FOA). Six different nonlinear functions are selected as testing functions. The experimental results illustrate that FOA cannot solve complex optimization problems effectively. In order to enhance the performance of FOA, an improved FOA (named LGMS-FOA) is proposed. Simulation results and comparisons of LGMS-FOA with FOA and other metaheuristics show that LGMS-FOA can greatly enhance the searching efficiency and greatly improve the searching quality.

In another paper, Y. Wang et al. present "Robust quadratic regression and its application to energy-growth consumption problem." The paper proposes a robust quadratic regression model to handle the statistics inaccuracy. Unlike the traditional robust statistic approaches that mainly focus on eliminating the effect of outliers, the proposed model employs the recently developed robust optimization methodology and tries to minimize the worst-case residual errors. First, they give a solvable equivalent semidefinite programming for the robust least square model with ball uncertainty set. Then the result is generalized to robust models under $l_{1}$ - and $l_{\infty}$-norm criteria with general ellipsoid uncertainty sets. In addition, they establish a robust regression model for per capita GDP and energy consumption in the energy-growth problem under the conservation hypothesis. Finally, numerical experiments are carried out to verify the effectiveness of the proposed models and demonstrate the effect of the uncertainty perturbation on the robust models.

In another paper, the research of L. Wang entitled "Extraction of belief knowledge from a relational database for quantitative Bayesian network inference," proposes the integration of a Bayesian network (BN) with the functional dependency (FD) discovery technique based on a three-phase learning framework. Association rule analysis is employed to discover FDs and expert knowledge encoded within a BN; that is, key relationships between attributes are emphasized. Moreover, the $\mathrm{BN}$ can be updated by using an expert-driven annotation process wherein redundant nodes and edges are removed. Experimental results show the effectiveness and efficiency of the proposed approach.

In the paper "Identification of code-switched sentences and words using language modeling approaches," L.-C. Yu et al. propose a language modeling approach to the problem of code-switching language processing, dividing the problem into two subtasks: the detection of code-switched sentences and the identification of code-switched words in sentences. A code-switched sentence is detected on the basis of whether it contains words or phrases from another language. Once the code-switched sentences are identified, the positions of the code-switched words in the sentences are then identified. Experimental results show that the language modeling approach achieved an F-measure of $80.43 \%$ and an accuracy of $79.01 \%$ for detecting Mandarin-Taiwanese code-switched sentences. For the identification of code-switched words, the word-based and POS-based models achieved F-measures of $41.09 \%$ and $53.08 \%$, respectively.

In the paper entitled "Knowledge mining based on environmental simulation applied to wind farm power forecasting," D. Niu et al. propose a self-organizing map (SOM) combined with rough set theory clustering technique (RST) to extract the relative knowledge and to choose the most similar history situation and efficient data for wind power forecasting with numerical weather prediction (NWP). Through integrating the SOM and RST methods to cluster the historical data into several classes, the approach could find the similar days and excavate the hidden rules. According to the data reprocessing, the selected samples will improve the forecast accuracy echo state network (ESN) trained by the class of the forecasting day that is adopted to forecast the wind power output accordingly. The developed methods are applied to a case of power forecasting in a wind farm located in northwest of China with wind power data from April 1, 2008, to May 6, 2009. In order to verify its effectiveness, the performance of the proposed method is compared with the traditional backpropagation neural network (BP). The results demonstrated that knowledge mining led to a promising improvement in the performance for wind farm power forecasting.

In the paper entitled "Matching cost filtering for dense stereo correspondence," Y. Lin et al. propose a new costaggregation module to compute the matching responses for all the image pixels at a set of sampling points generated by a hierarchical clustering algorithm. The complexity of this implementation is linear both in the number of image pixels and in the number of clusters. Experimental results demonstrate that the proposed algorithm outperforms stateof-the-art local methods in terms of both accuracy and speed. Moreover, performance tests indicate that parameters such as the height of the hierarchical binary tree and the spatial and range standard deviations have a significant influence on time consumption and the accuracy of disparity maps.

In the paper entitled "A multilayer hidden Markov modelsbased method for human-robot interaction," C. Tao and G. Liu propose a continuous gesture recognition approach based on multilayer hidden Markov models (MHMMs) to achieve human-robot interaction (HRI) by using gestures. The method consists of two parts. One part is gesture spotting and segment module, the other part is continuous gesture recognition module. Firstly, a Kinect sensor is used to capture 3D acceleration and 3D angular velocity data of hand gestures. Then, feed-forward neural networks (FNNs) and a threshold criterion are used for gesture spotting and segment, respectively. Afterwards, the segmented gesture signals are, respectively, preprocessed and vector symbolized by a sliding window and a K-means clustering method. Finally, symbolized data are sent into lower hidden Markov models (LHMMs) to identify individual gestures, and, then, a Bayesian filter with sequential constraints among gestures in upper hidden Markov models (UHMMs) is used to correct recognition errors created in LHMMs. Five predefined gestures are used to interact with a Kinect mobile robot in experiments. The experimental results show that the proposed method not only has good effectiveness and accuracy, but also has favorable real-time performance.

In the paper entitled "Nonlinear predictive control of mass moment aerospace vehicles based on ant colony genetic algorithm optimization," X. Zhang et al. propose a novel kind of NPC parameters optimization strategy based on ant colony genetic algorithm (ACGA), aiming at the parameters of NPC that is generally used the trial-and-error method to optimize 
and design. The method for setting NPC parameters with ACA in which the routes of ants are optimized by the genetic algorithm (GA) is derived. And then, a detailed realized process of this method is also presented. Furthermore, this optimization algorithm of the NPC parameters is applied to the flight control system of MMAV. The simulation results show that the system not only meets the demands of timeresponse specifications but also has excellent robustness.

In the paper entitled "Modeling and analysis of the weld bead geometry in submerged arc welding by using adaptive neurofuzzy inference system," N. Akkas et al. aim at obtaining a relationship between the values defining bead geometry and the welding parameters and also to select optimum welding parameters. For this reason, an experimental study has been realized. The welding parameters such as the arc current, arc voltage, and welding speed that have the most effect on bead geometry are considered, and the other parameters are held as constant. Four, three, and five different values for the arc current, the arc voltage, and welding speed are used, respectively. Therefore, sixty samples made of St 52-3 material are prepared. The bead geometries of the samples are analyzed, and the thickness and penetration values of the weld bead are measured. Then, the relationship between the welding parameters is modeled by using artificial neural network (ANN) and neurofuzzy system approach. Each model is checked for its adequacy by using test data that are selected from experimental results. Then, the models developed are compared with regard to accuracy. In addition, the appropriate welding parameters values can be easily selected when the models improve.

In the paper entitled "Genetic pattern search and its application to brain image classification," Y. Zhang et al. propose a novel global optimization method based on the combination of genetic algorithm (GA) and generalized pattern search (PS) algorithm, to find global minimal points more effectively and rapidly. The idea lies in the facts that GA tends to be quite good at finding generally good global solutions but quite inefficient in finding the last few mutations for the absolute optimum and that PS is quite efficient in finding absolute optimum in a limited region. The novel algorithm, named as genetic pattern search (GPS), employs the GA as the search method at every step of PS. Experiments on five different classical benchmark functions (consisting of Hump, Powell, Rosenbrock, Schaffer, and Woods) demonstrate that the proposed GPS is superior to improved GA and improved PS with respect to success rate. They apply the GPS to the classification of normal and abnormal structural brain MRI images. The results indicate that GPS exceeds BP, MBP, IGA, and IPS in terms of classification accuracy. This suggests that GPS is an effective and viable global optimization method and can be applied to brain MRI classification.

In the paper entitled "Solving a novel inventory location model with stochastic constraints and $(R, s, S)$ inventory control policy," G. Cabrera et al. solve a novel inventorylocation model with a stochastic capacity constraint based on a periodic inventory control (ILM-PR) policy. The ILM-PR policy implies several changes with regard to other previous models proposed in the literature, which consider continuous review as their inventory policy. One of these changes is the inclusion of the undershoot concept, which has not been considered in previous ILM models in the literature. Based on the model, they design a distribution network for a two-level supply chain, addressing both warehouse location and customer assignment decisions, whilst taking into consideration several aspects of inventory planning, in particular, evaluating the impact of the inventory control review period on the network configuration and system costs. Because the model is a very hard-to solve combinatorial nonlinear optimization problem, they implemented two heuristics to solve it, namely, Tabu Search and Particle Swarm Optimization. These approaches are tested over small instances in which they are able to find the optimal solution in just a few seconds. Because the model is a new one, a set of medium-size instances is provided that can be useful as a benchmark in future research. The heuristics show a good convergence rate when applied to those instances. The results confirm that decision making over the inventory control policy has effects on the distribution network design.

In another paper, S. Dinç et al. present "Hyperspectral image classification using kernel Fukunaga-Koontz transform." The paper presents a novel approach for the hyperspectral imagery (HSI) classification problem, using Kernel Fukunaga-Koontz transform (K-FKT). The Kernel based Fukunaga-Koontz transform offers higher performance for classification problems due to its ability to solve nonlinear data distributions. K-FKT is realized in two stages: training and testing. In the training stage, unlike classical FKT, samples are relocated to the higher dimensional kernel space to obtain a transformation from nonlinear distributed data to linear form. This provides a more efficient solution to hyperspectral data classification. The second stage, testing, is accomplished by employing the Fukunaga-Koontz transformation operator to find out the classes of the real world hyperspectral images. In experiment section, the improved performance of HSI classification technique, K-FKT, has been tested comparing other methods such as the classical FKT and three types of support vector machines (SVMs).

In another paper, the research of A. El Mobacher et al. entitled "Entropy-based and weighted selective SIFT clustering as an energy aware framework for supervised visual recognition of man-made structures," presents uSee, a supervised learning framework which exploits the symmetrical and repetitive structural patterns in buildings to identify subsets of relevant clusters formed by these keypoints. Once a smart phone captures an image, uSee preprocesses it using variations in gradient angle- and entropy-based measures before extracting the building signature and comparing its representative SIFT keypoints against a repository of building images. Experimental results on 2 different databases confirm the effectiveness of uSee in delivering, at a greatly reduced computational cost, the high matching scores for building recognition that local descriptors can achieve. With only $14.3 \%$ of image SIFT keypoints, uSee exceeded prior literature results by achieving an accuracy of $99.1 \%$ on the Zurich Building Database with no manual rotation, thus saving significantly on the computational requirements of the task at hand. 
In the paper "Application of adaptive extended Kalman smoothing on INS/WSN integration system for mobile robot indoors," X. Chen et al. propose the inertial navigation systems (INS)/wireless sensor network (WSN) integration system of mobile robot for navigation information indoors accurately and continuously. The Kalman filter (KF) is widely used for real-time applications with the aim of gaining optimal data fusion. In order to improve the accuracy of the navigation information, they propose an adaptive extended Kalman smoothing (AEKS) which utilizes inertial measuring units (IMUs) and ultrasonic positioning system. The adaptive extended Kalman filter (AEKF) is used to improve the accuracy of forward Kalman filtering (FKF) and backward Kalman filtering (BKF), and then the AEKS and the average filter are used between two output timings for the online smoothing. Several real indoor tests are done to assess the performance of the proposed method. The results show that the proposed method can reduce the error compared with the INS-only, least squares (LS) solution, and AEKF.

In the paper entitled "The study of reinforcement learning for traffic self-adaptive control under multiagent Markov game environment," L.-H. Xu et al. cast traffic self-adaptive control as a multiagent Markov game problem. The design employs traffic signal control agent (TSCA) for each signalized intersection that coordinates with neighboring TSCAs. A mathematical model for TSCAs' interaction is built based on nonzero-sum Markov game that has been applied to let TSCAs learn how to cooperate. A multiagent Markov game reinforcement learning approach is constructed on the basis of single-agent Q-learning. Their method lets each TSCA learn to update its Q-values under the joint actions and imperfect information. The convergence of the proposed algorithm is analyzed theoretically. The simulation results show that the proposed method is convergent and effective in realistic traffic self-adaptive control setting.

In the paper entitled "Research on ISFLA-based optimal control strategy for the coordinated charging of EV battery swap station," X. Huang et al. maintain that electric vehicles (EVs) could be a good measure against energy shortages and environmental pollution as an important component of the smart grid. A main way of energy supply to EVs is to swap battery from the swap station. Based on the characteristics of EV battery swap station, the coordinated charging optimal control strategy is investigated to smooth the load fluctuation. Shuffled frog leaping algorithm (SFLA) is an optimization method inspired by the memetic evolution of a group of frogs when seeking food. An improved shuffled frog leaping algorithm (ISFLA) with the reflecting method to deal with the boundary constraint is proposed to obtain the solution of the optimal control strategy for coordinated charging. Based on the daily load of a certain area, the numerical simulations including the comparison of PSO and ISFLA are carried out and the results show that the presented ISFLA can effectively lower the peak-valley difference and smooth the load profile with the faster convergence rate and higher convergence precision.

In the paper entitled "Composite broadcasting and ranging via a satellite dual-frequency MPPSK system," Y. Yao et al. propose the design of dual frequency M-ray position phase shift keying (MPPSK) system that is suitable for performing both data transmission and range measurement. The approach is based on MPPSK modulation waveforms utilized in digital video broadcasting. In particular, requirements that allow for employing such signals for range measurements with high accuracy and high range are investigated. In addition, the relationship between the frequency difference of dual frequency MPPSK system and range accuracy is discussed. Moreover, the selection of MPPSK modulation parameter for data rate and ranging is considered. In addition to theoretical considerations, the paper presents system simulations and measurement results of new systems, demonstrating the high spectral utilization of integrated broadcasting and ranging applications.

In the paper entitled "A genetic-algorithms-based approach for programming linear and quadratic optimization problems with uncertainty," W. Jin et al. propose a geneticalgorithms-based approach as an all-purpose problemsolving method for operation programming problems under uncertainty. The proposed method is applied for management of a municipal solid waste treatment system. Compared to the traditional interactive binary analysis, this approach has fewer limitations and is able to reduce the complexity in solving the inexact linear programming problems and inexact quadratic programming problems. The implementation of this approach is performed using the genetic algorithm solver of MATLAB (trademark of MathWorks). The paper explains the genetic-algorithmsbased method and presents details on the computation procedures for each type of inexact operation programming problems. A comparison of the results generated by the proposed method based on genetic algorithms with those produced by the traditional interactive binary analysis method is also presented.

In the paper entitled "Solving the balanced academic curriculum problem using the ACO metaheuristic," J.-M. Rubio et al. consider that the balanced academic curriculum problem consists in the assignation of courses to academic periods satisfying all the load limits and prerequisite constraints. They present the design of a solution to the balanced academic curriculum problem based on the ACO metaheuristic, in particular via the best-worst ant system. They provide an experimental evaluation that illustrates the effectiveness of the proposed approach on a set of classic benchmarks as well as on real instances.

In the paper entitled "Hybrid functional-neural approach for surface reconstruction,” A. Iglesias and A. Gálvez introduce a new hybrid functional-neural approach for surface reconstruction. The approach is based on the combination of two powerful artificial intelligence paradigms: on one hand, they apply the popular Kohonen neural network to address the data parameterization problem. On the other hand, they introduce a new functional network, called NURBS functional network, whose topology is aimed at reproducing faithfully the functional structure of the NURBS surfaces. These neural and functional networks are applied in an iterative fashion for further surface refinement. The hybridization of these two networks provides a powerful computational approach to obtain a NURBS fitting surface 
to a set of irregularly sampled noisy data points within a prescribed error threshold. The method has been applied to two illustrative examples. The experimental results confirm the good performance of their approach.

In the paper entitled "An algorithm for mining of association rules for the information communication network alarms based on swarm intelligence," Y. Wang et al. analyze the data characteristics and association logic of the network alarms. Besides, the alarm data are preprocessed and the main standardization information fields are screened. The APPSO algorithm is proposed on the basis of combining the evaluation method for support and confidence coefficient in the Apriori (AP) algorithm as well as the particle swarm optimization (PSO) algorithm. By establishing a sparse linked list, the algorithm is able to calculate the particle support thus further improving the performance of the APPSO algorithm. Based on the test for the network alarm data, it is discovered that rational setting of the particle swarm scale and number of iterations of the APPSO algorithm can be used to mine the vast majority and even all of the association rules and the mining efficiency are significantly improved, compared with Apriori algorithm.

In another paper, S.-F. Jiang et al. present "Structural reliability assessment by integrating sensitivity analysis and support vector machine." This paper proposes a structural reliability assessment method by the use of sensitivity analysis (SA) and support vector machine (SVM). The sensitivity analysis is firstly applied to assess the effect of random variables on the values of performance function, while the smallinfluence variables are rejected as input vectors of SVM. Then, the trained SVM is used to classify the input vectors, which are produced by sampling the residual variables based on their distributions. Finally, the reliability assessment is implemented with the aid of reliability theory. A 10-bar planar truss is used to validate the feasibility and efficiency of the proposed method, and a performance comparison is made with other existing methods. The results show that the proposed method can largely save the runtime with less reduction of the accuracy; furthermore, the accuracy using the proposed method is the highest among the methods employed.

In a paper, the research of C. Liu et al. entitled "Incremental tensor principal component analysis for handwritten digit recognition" proposes incremental tensor principal component analysis (ITPCA) based on updated-SVD technique algorithm. This paper proves the relationship between PCA, 2DPCA, MPCA, and the graph embedding framework theoretically and derives the incremental learning procedure to add single sample and multiple samples in detail. The experiments on handwritten digit recognition have demonstrated that ITPCA has achieved better recognition performance than that of vector-based principal component analysis (PCA), incremental principal component analysis (IPCA), and multilinear principal component analysis (MPCA) algorithms. At the same time, ITPCA also has lower time and space complexity.

In the paper "Optimum performance-based seismic design using a hybrid optimization algorithm," S. Talatahari et al. present a hybrid optimization method to optimum seismic design of steel frames considering four performance levels. These performance levels are considered to determine the optimum design of structures to reduce the structural cost. A pushover analysis of steel building frameworks subject to equivalent-static earthquake loading is utilized. The algorithm is based on the concepts of the charged system search in which each agent is affected by local and global best positions stored in the charged memory considering the governing laws of electrical physics. Comparison of the results of the hybrid algorithm with those of other metaheuristic algorithms shows the efficiency of the hybrid algorithm.

In the paper entitled "Research on cooperative combat for integrated reconnaissance-attack-BDA of group LAVs," L. Bing et al. analyze a group system by the theory of Itô stochastic differential. The uniqueness and continuity of the solution of the system are discussed. Afterwards the model of the system based on the state transition is established with the finite state machine automatically. At last, a search algorithm is proposed for obtaining good feasible solutions for problems. Moreover, simulation results show that model and method are effective for dealing with cooperative combat of group LAVs (loitering air vehicles).

In the paper entitled "Wolf pack algorithm for unconstrained global optimization," H.-S. Wu and F.-M. Zhang abstract three intelligent behaviors, scouting, calling, and besieging, and two intelligent rules, winner-take-all generation rule of lead wolf and stronger-survive renewing rule of wolf pack. Then they propose a new heuristic swarm intelligent method, named wolf pack algorithm (WPA). Experiments are conducted on a suit of benchmark functions with different characteristics, unimodal/multimodal, separable/nonseparable, and the impact of several distance measurements and parameters on WPA are discussed. What is more, the compared simulation experiments with other five typical intelligent algorithms, genetic algorithm, particle swarm optimization algorithm, artificial fish swarm algorithm, artificial bee colony algorithm, and firefly algorithm, show that WPA has better convergence and robustness, especially for high-dimensional functions.

In the paper entitled "Towards a unified sentiment lexicon based on graphics processing units," L. I. Barbosa-Santillán et al. present an approach to create what they have called a unified sentiment lexicon (USL). This approach aims at aligning, unifying, and expanding the set of sentiment lexicons that are available on the web in order to increase their robustness of coverage. One problem related to the task of the automatic unification of different scores of sentiment lexicons is that there are multiple lexical entries for which the classification of positive, negative, or neutral $(P, N, Z)$ depends on the unit of measurement used in the annotation methodology of the source sentiment lexicon. Their USL approach computes the unified strength of polarity of each lexical entry based on the Pearson correlation coefficient that measures how correlated lexical entries are with a value between 1 and -1 , where 1 indicates that the lexical entries are perfectly correlated, 0 indicates no correlation, and -1 means they are perfectly inversely correlated and so is the unified metrics procedure for CPU and GPU, respectively. Another problem is the high processing time required for computing 
all the lexical entries in the unification task. Thus, the USL approach computes a subset of lexical entries in each of the 1344 GPU cores and uses parallel processing in order to unify 155802 lexical entries. The results of the analysis conducted using the USL approach show that the USL has 95.430 lexical entries, out of which there are 35.201 considered positive, 22.029 negative, and 38.200 neutral. Finally, the runtime is 10 minutes for 95.430 lexical entries; this allows a reduction of the time computing for the unified metrics by 3 times.

In the paper entitled "The application of FastICA combined with related function in blind signal separation," D. Li et al. propose a new method which is the combination of related function relevance to estimated signal and negative entropy in fast independent component analysis (FastICA) as objective function, and the iterative formula is derived without any assumptions, then the independent components are found by maximizing the objective function. The improved algorithm shorthand for R-FastICA is applied to extract random mixed signals and ventricular late potential (VLP) signal from normal ECG signal; simultaneously the performance of R-FastICA algorithm is compared with traditional FastICA through simulation. Experimental results show that R-FastICA algorithm outperforms traditional FastICA with higher similarity coefficient and separation precision.

\section{Acknowledgments}

We would like to express our gratitude to all of the authors for their contributions and the reviewers for their effort providing constructive comments and feedback. We hope this special issue offers a comprehensive and timely view of the area of emerging trends in artificial intelligence and its applications and that it will offer stimulation for further research.

Yudong Zhang

Saeed Balochian

Praveen Agarwal

Vishal Bhatnagar

Orwa Jaber Housheya 


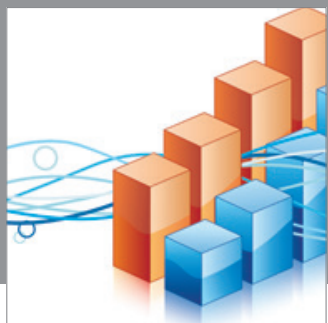

Advances in

Operations Research

mansans

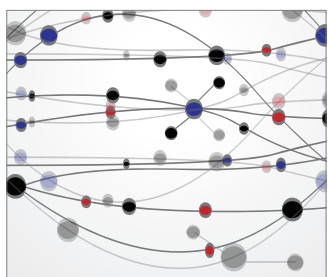

The Scientific World Journal
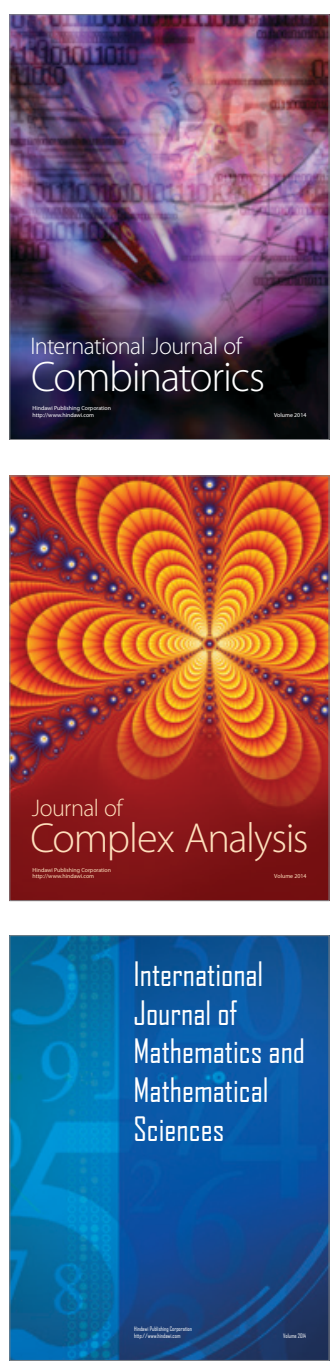
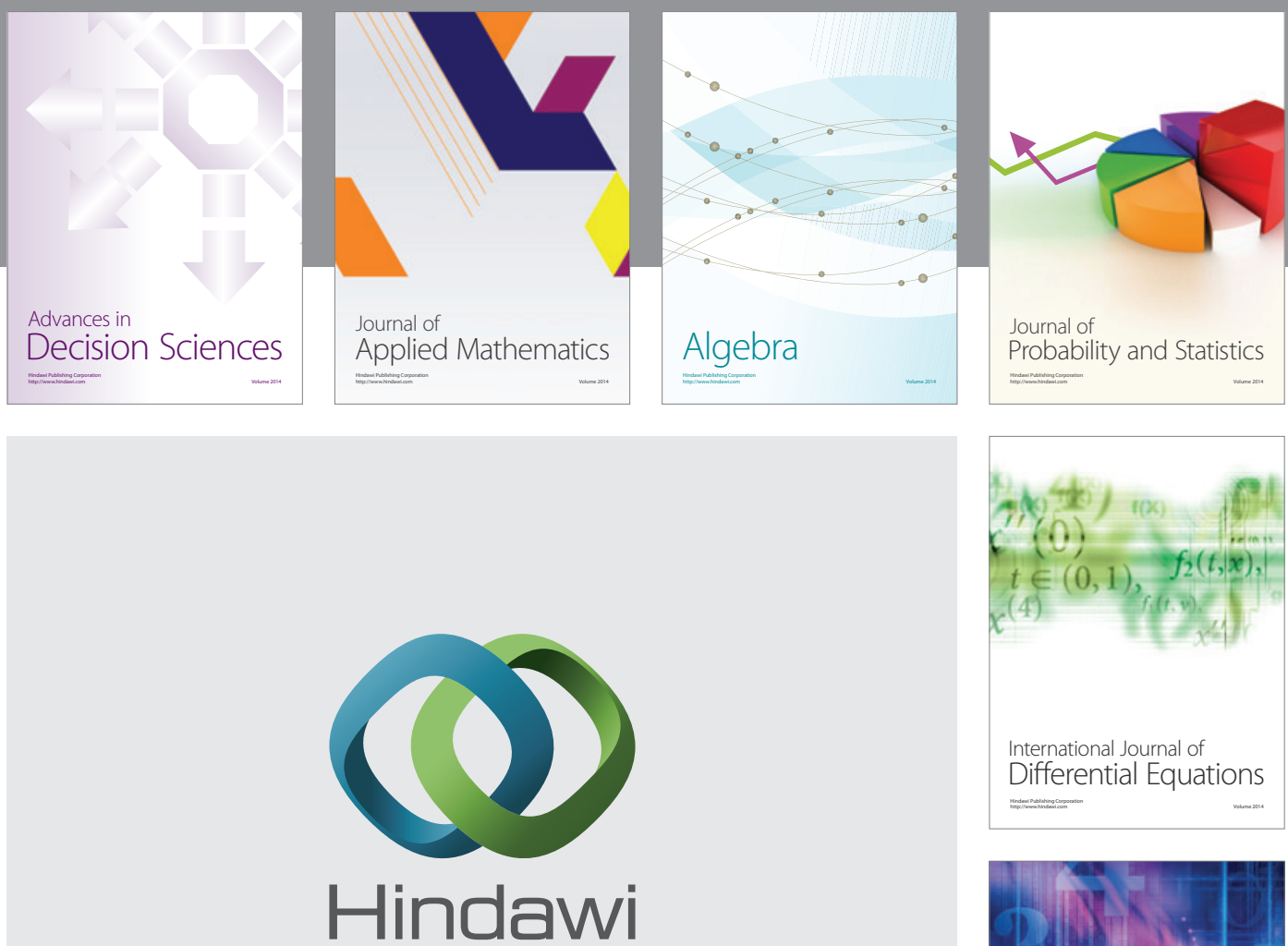

Submit your manuscripts at http://www.hindawi.com
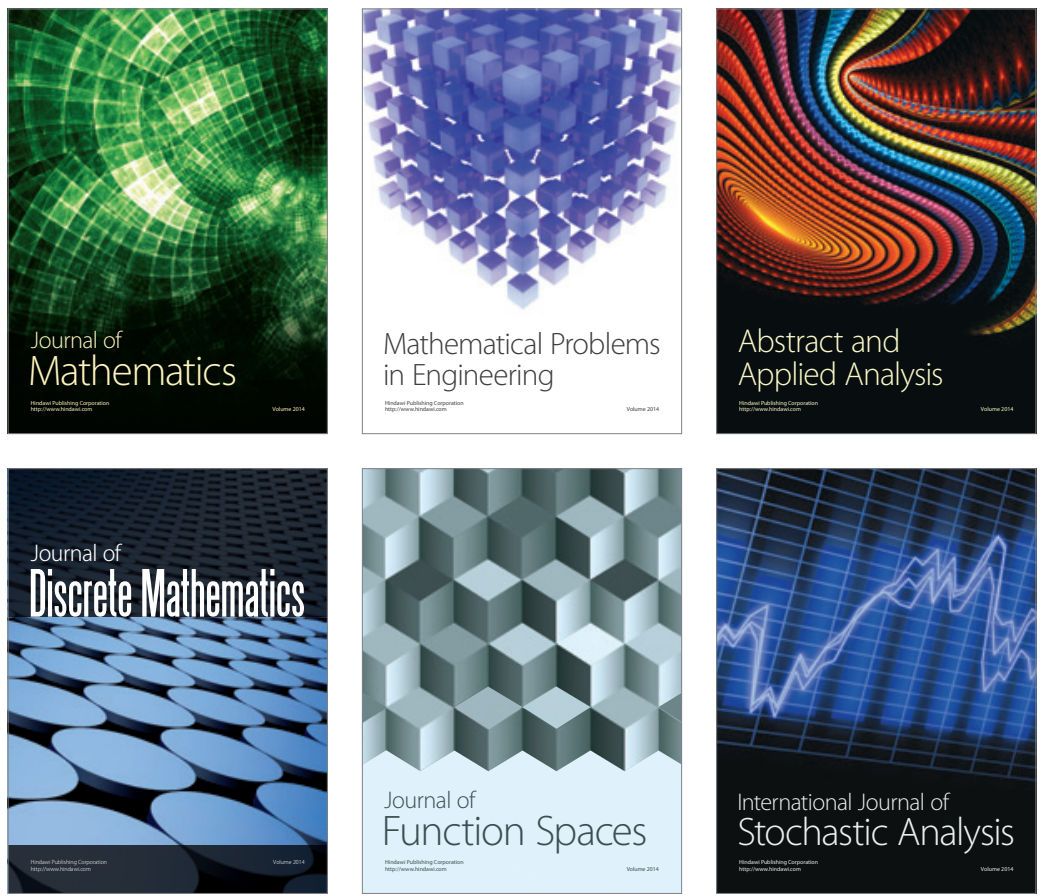

Journal of

Function Spaces

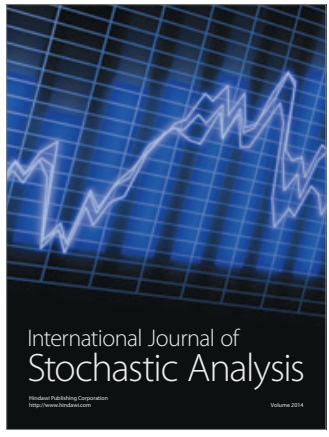

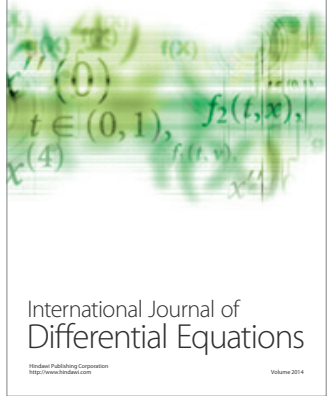
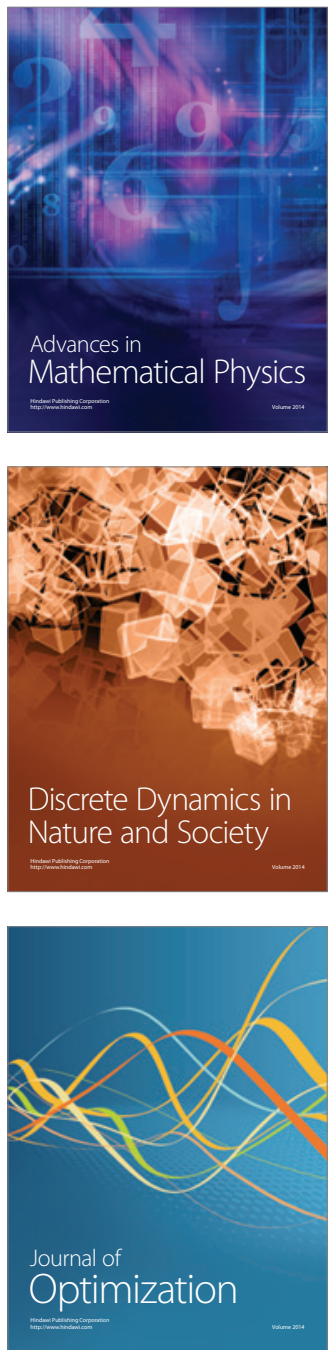